\title{
Über die Metallverbindungen des Pyridins und die Elektrolyse des Pyridins.
}

\author{
Von \\ Ludwig Pincussohn.
}

Die ersten genaueren Angaben über Pyridinsalze stammen aus dem Jahre 1858 von Th. Anderson, ${ }^{1}$ dem Entdecker des Pyridins. Er fand, dafs das Pyridin grofse Neigung habe, Doppelsalze zu bilden, die meist leicht krystallisierten und das Metalloxyd in einem solchen Zustande enthielten, dafs es durch überschüssiges Pyridin nicht gefällt wurde.

Ein Golddoppelsalz erhielt er durch Zusatz von Goldchlorid zu einer Lösung von salzsaurem Pyridin als citronengelbes, krystallinisches Pulver, das sich aus heilsem Wasser in gelben Nadeln umkrystallisieren liefs. Das Salz entsprach der Formel $\mathrm{C}_{5} \mathrm{H}_{5} \mathrm{~N} \cdot \mathrm{HCl} \cdot \mathrm{AuCl}_{3}$. Wurde Pyridin in beträchtlichem Überschufs zu Zinksulfat gesetzt, so erfolgten Abscheidungen von Zinkoxyd. Bei Zusatz einer zur Neutralisation ungenügenden Menge von Salzsäure erfolgte Klarwerden der Flüssigkeit; durch rasches Umrühren fiel ein Doppelsalz aus. Auch Kupfersulfat bildete ein Doppelsalz. Dals auch Mangan, Nickel, Eisenoxydul Doppelsalze bilden dürften, wird von ANDERson erwähnt, näher untersucht hat er diese Salze nicht.

Ein Platindoppelsalz der Formel $\mathrm{C}_{10} \mathrm{H}_{5} \mathrm{~N} . \mathrm{HCl} . \mathrm{PtCl}_{2}$ erwähnt Anderson bereits in einer Arbeit des Jahres 1855: „Vorläufige Notiz über die Zersetzung der Platindoppelsalze der organischen Säuren.“ 2

Im Jahre 1883 beschrieb dann S. M. JönGENSEN ${ }^{3}$ verschiedene Rhodiumverbindungen, denen er Formeln wie $\mathrm{Cl}_{4}\left[\mathrm{Rh}_{2} \cdot 8 \mathrm{C}_{5} \mathrm{H}_{5} \mathrm{~N}\right] \mathrm{Cl}_{2}$, $\mathrm{Cl}_{4}\left[\mathrm{Rh}_{2} \cdot 8 \mathrm{C}_{5} \mathrm{H}_{5} \mathrm{~N}\right] \cdot 2 \mathrm{NO}_{3}, \mathrm{Cl}_{4}\left[\mathrm{Rh}_{2} \cdot 8 \mathrm{C}_{5} \mathrm{H}_{5} \mathrm{~N}\right] \mathrm{SO}_{4}$ unterlegt. Im Jahre

\footnotetext{
1 Transact. Roy. Soc. Edinbourgh [4] 21, 571.

2 Lieb. Ann. 96, 199.

${ }^{3}$ Journ. pr. Chem. (1885) [2] 27, 478.
} 
1886 beschrieb derselbe ${ }^{1}$ verschiedene Platinopyridinsalze. Für solche Salze stellte er z. B. die folgenden Formeln auf:

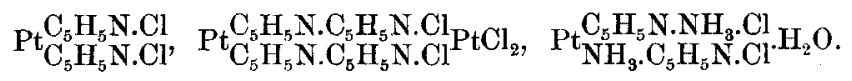

Auch Silber- und Kupfersalze sind von JöRGEnsEN dargestellt und analysiert worden. ${ }^{2}$ Aufser dem Salz der Formel (Ag.2 $\left.\mathrm{C}_{5} \mathrm{H}_{5} \mathrm{~N}\right) \mathrm{NO}_{3}$ wurde noch ein anderes (Ag. $3 \mathrm{C}_{5} \mathrm{H}_{5} \mathrm{~N}_{2} \mathrm{NO}_{3}$ erhalten, das sich als unbeständig erwies und leicht in das erstere überging. Von Kupfersalzen nennt JöRGENSEN ein Cupriddipyridinsulfat $\left(\mathrm{Cu} .4 \mathrm{C}_{5} \mathrm{H}_{5} \mathrm{~N}\right) \mathrm{SO}_{4}$, und das Cupriddipyridindithionat $\left(\mathrm{Cu} .4 \mathrm{C}_{5} \mathrm{H}_{5} \mathrm{~N}\right) \mathrm{S}_{2} \mathrm{O}_{6}$.

W. KöNIGS und R. GEIGY ${ }^{3}$ erwähnen qualitativ einige Metallsalze des Pyridins. Nach ihren Angaben giebt Pyridin selbst in stark verdünnter wässeriger Lösung mit Quecksilberchlorid eine krystallisierende Verbindung, die sich in kochendem Wasser löst und beim Erkalten in langen weifsen Nadeln ausscheidet; ebenso entsteht bei derselben Verdünnung mit Cadmiumchlorid nach mehrstündigem Stehen eine krystallisierende Verbindung, die sich in Bezug auf Löslichkeit in Wasser wie die Quecksilberverbindung verhält. Ebenso mit Quecksilberjodid. Sehr schwer löslich ist der Niederschlag, den Phosphorwolframsäure in einer sauren Lösung von Pyridin hervorruft. Analysen wurden nicht ausgeführt.

Doppelsalze aus Metallsalz und Pyridinsalz stellte Monari ${ }^{4}$ her. Aus Quecksilberchlorid und salzsaurem Pyridin erhielt er ein Doppelsalz der Formel $\mathrm{C}_{5} \mathrm{H}_{5} \mathrm{~N} . \mathrm{HCl} .2 \mathrm{HgCl}_{2}$. Aus Pyridin und Quecksilberchlorid erhielt er $\mathrm{C}_{5} \mathrm{H}_{5} \mathrm{~N} .3 \mathrm{HgCl}_{2}$, und entsprechend $\left(\mathrm{C}_{5} \mathrm{H}_{5} \mathrm{~N}_{2} \mathrm{CdJ}_{2}\right.$ und $\left(\mathrm{C}_{5} \mathrm{H}_{5} \mathrm{~N}\right)_{2} \cdot \mathrm{ZnCl}_{2}$.

WilHelm Lang ${ }^{5}$ teilt die anorganischen Basen nach ihrem Verhalten zu Pyridin in zwei Teile. Aus der wässerigen Lösung der einen wie Eisenchlorid, Eisenoxydulsulfat, Aluminiumsulfat, Chromchlorid, Chromisulfat wird das Metall durch Pyridin als Hydroyxd gefällt; die Mehrzahl der übrigen Metalle bildet mit Pyridin Doppelverbindungen.

Unter den Salzen, die LaNg erhielt, ist zuerst das Zinkchloridpyridin zu nennen $\left(\mathrm{ZnCl}_{2} \cdot 2 \mathrm{C}_{5} \mathrm{H}_{5} \mathrm{~N}\right)$. Beim Versetzen der wässerigen

\footnotetext{
1 Journ. pr. Chem. (1886) [2] 33, 504.

2 1. c. S. 502 .

3 Ber. deutsch. chem. Qes. (1884) 17, 594.

4 Rev. chim. med. farm. 2, 190.

s Ber. deutsch. chem. Ges. (1888) 21, 1578.
} 
Lösung von Zinkchlorid mit einem Überschufs von Pyridin entstand ein Niederschlag, der aus siedendem, pyridinhaltigem Wasser in Centimeter langen, weilsen, seideglänzenden Nadeln krystallisierte, die sich unter dem Mikroskop als faserige Aggregate erwiesen. Das Salz löste sich auch in siedendem Alkohol und krystallisierte bei ganz langsamem Erkalten in kurzen glasglänzenden Prismen mit domatischen oder pinakoidalen Flächen. Wurde das Salz in Chlorwasserstoffsäure gelöst, so bildete sich beim Eindampfen das schwach gelblich gefärbte Doppelsalz $\mathrm{ZnCl}_{2} \cdot 2\left(\mathrm{C}_{5} \mathrm{H}_{5} \mathrm{~N} . \mathrm{HCl}\right)$, welches in kaltem Alkohol schwer löslich war.

Kupferchloridpyridin $\left(\mathrm{CuCl}_{2} \cdot 2 \mathrm{C}_{5} \mathrm{H}_{5} \mathrm{~N}\right)$ wurde durch Versetzen der alkoholischen Lösung von Kupferchlorid mit Pyridin erhalten. Grünblaue, seidenglänzende Nadeln. Schmelzpunkt $185^{\circ}$. Beim Eindampfen färbte sich die Flüssigkeit grün; es hinterblieben dunkelgelbe Prismen des Doppelsalzes $\mathrm{CuCl}_{2}\left(2 \mathrm{C}_{5} \mathrm{H}_{6} \mathrm{~N} . \mathrm{HCl}\right)$, das aus Wasser umkrystallisiert werden konnte, beim Auflösen in siedendem Alkohol aber wieder in das einfache $\mathrm{Salz}\left(\mathrm{CuCl}_{2}, 2 \mathrm{C}_{5} \mathrm{H}_{5} \mathrm{~N}\right)$ zurückverwandelt wurde.

Kupfersulfatpyridin $\left(\mathrm{CuSO}_{4} \cdot \mathrm{C}_{5} \mathrm{H}_{5} \mathrm{~N}_{3} 3 \mathrm{H}_{2} \mathrm{O}\right)$. LaNG versetzte die wässerige Lösung von Kupfersulfat mit wenig Pyridin und erhielt einen Niederschlag, der sich in überschüssigem Pyridin mit tiefblauer Farbe löste. Beim Schütteln fiel das Doppelsalz in blauen mikroskopischen Nadeln aus. Aus Alkohol krystallisierte es in schönen himmelblauen Nadeln von starkem Seidenglanz.

Kupferchlorürpyridin erhielt $\mathrm{L}_{\mathrm{ANG}}$ in zwei Modifikationen mit verschiedenem Pyridingehalt: $\mathrm{Cu}_{2} \mathrm{Cl}_{2} \cdot 4 \mathrm{C}_{5} \mathrm{H}_{5} \mathrm{~N}$ und $\mathrm{Cu}_{2} \mathrm{Cl}_{2} \cdot 6 \mathrm{C}_{5} \mathrm{H}_{5} \mathrm{~N}$.

Aus einer alkoholischen Lösung von Cadmiumchlorid wurden mit Pyridin Nadeln von Cadmiumchloridpyridin gefällt. Die Verbindung $\left(\mathrm{CdCl}_{2}, 2 \mathrm{C}_{5} \mathrm{H}_{5} \mathrm{~N}\right)$ krystallisierte in charakteristischen Nadeln.

Aus einer wässerigen Lösung von Cadmiumjodid erhielt er $\mathrm{CdJ}_{2} \cdot 2 \mathrm{C}_{5} \mathrm{H}_{5} \mathrm{~N}$.

Die Mehrzahl der genannten Salze ist nach LaNG's Angaben sehr beständig und giebt das Pyridin verhältnismäfsig schwer ab. So verliert z. B. Kupfersulfatpyridin in 9 Stunden bei $100^{\circ}$ nur eine Spur Pyridin, bei $120-130^{\circ}$ in. 2 Stunden $5.6 \%$, in derselben Zeit bei $140-150^{\circ} 17.4 \%$ Pyridin. LANG stellte noch ein Quecksilberchloridpyridin $\mathrm{HgCl}_{2} \cdot \mathrm{C}_{5} \mathrm{H}_{5} \mathrm{~N}$ her, ein entsprechendes Nitrat der Formel $\mathrm{Hg}\left(\mathrm{NO}_{3}\right)_{2} \mathrm{C}_{5} \mathrm{H}_{5} \mathrm{~N}$; ferner Verbindungen des Calciumchlorids, Kobaltchlorürs, des Ferrosulfates und des Nickelsulfates mit Pyridin.

z. anorg. Chem, XIV. 
Ladendurg ${ }^{1}$ (Über Pyridin- und Piperidinbasen) beschrieb ausführlich das schon erwähnte Quecksilberchlorid-Pyridinchlorhydrat $\left(\mathrm{C}_{5} \mathrm{H}_{5} \mathrm{~N} . \mathrm{HCl} .2 \mathrm{HgCl}_{2}\right)$, das er zur Reinigung und Charakterisierung des Pyridins als sehr geeignet empfiehlt. Ein hergestelltes Platindoppelsalz war in kaltem Wasser schwer, in heilsem leicht löslich, ein Golddoppelsalz - kleine Prismen - war auch in heifsem Wasser schwer in Lösung zu bringen.

Ein Quecksilbersalz der Formel $\mathrm{C}_{5} \mathrm{H}_{6} \mathrm{~N} \cdot \mathrm{HgCl}_{2}$ erhielt auch $\mathrm{Her}$ MaNN Thoms, ${ }^{2}$ und zwar nicht wie LaNG, aus wässeriger, sondern aus alkoholischer Pyridinlösung.

LACHOwICZ und $\mathrm{B}_{\triangle \mathrm{NDROWSKI}}{ }^{3}$ stellten ein Zinkdoppelsalz der Formel $\left(2 \mathrm{C}_{5} \mathrm{H}_{5} \mathrm{~N}\right) \cdot \mathrm{ZnCl}_{2}+2 \mathrm{H}_{2} \mathrm{O}$ her.

Arthur M. Comey und F. W. Smith ${ }^{4}$ (Silicotetrafluorides of certain bases) lielsen Siliciumtetrafluorid auf verschiedene Basen einwirken. Indem sie Siliciumtetrafluorid in eine Lösung von Pyridin in Benzol leiteten, erhielten sie als voluminösen Niederschlag, der mit Benzol gewaschen und dann getrocknet wurde, ein Salz der Formel $\left(\mathrm{C}_{5} \mathrm{H}_{5} \mathrm{~N}_{2} \mathrm{SiFl}_{4}\right.$. $\left(3 \mathrm{C}_{5} \mathrm{H}_{5} \mathrm{~N}\right.$. $)\left(\mathrm{SiFl}_{4}\right)_{2}$ wurde durch Sublimation des ersteren erhalten.

A. Classen und B. Zahorsky ${ }^{5}$ erhielten durch Umsetzung des gelben Ammonsalzes $2 \mathrm{PbCl}_{4} .5 \mathrm{NH}_{4} \mathrm{Cl}$ mit heil'ser Pyridinlösung gelbe Nadeln von Bleitetrachloridpyridinchlorhydrat $\left(2 \mathrm{PbCl}_{4} .5 \mathrm{C}_{5} \mathrm{H}_{5} \mathrm{~N} \cdot \mathrm{HCl}\right)$. Aufserdem wurden die Salze $3 \mathrm{PbCl}_{2}, 4 \mathrm{C}_{5} \mathrm{H}_{5} \mathrm{~N}$ und $3 \mathrm{PbCl}_{2}, 4 \mathrm{C}_{5} \mathrm{H}_{5} \mathrm{~N} . \mathrm{HCl}$ dargestellt.

Endlich hat noch Stanislaus v. Lascynski ${ }^{6}$ einige Pyridinsalze beschrieben: Lithiumchloridpyridin $\left(\mathrm{LiCl} .2 \mathrm{C}_{5} \mathrm{H}_{5} \mathrm{~N}\right)$ und Jodsilberpyridin.

Über die Elektrolyse des Pyridins hat im April 1896 Feurx B. AHRENs ${ }^{7}$ eine Arbeit veröffentlicht, deren Resultate auch schon in das vorher erschienene Lehrbuch desselben ${ }^{8}$ aufgenommen sind. Wird Pyridin in etwa der zehnfachen Menge 10\% iger Schwefelsäure gelöst und in einer von $10 \%$ iger Schwefelsäure umgebenen Thonzelle

1 Lieb. Ann. (1888) 247, 5.

- Pharm. Centralh. 29, 317-319.

${ }^{3}$ Monatsh. Chem. 9, 510-517.

4 Amer. chem. Journ. 10, 294.

${ }^{5}$ Diese Zeitschr. 4, 101-110.

- Inaug.-Diss. 1894.

7 Zeitschr. Elektrochemie (1896) 2, Heft 26.

s Ahress, Hundbuch der Elehtrochemie. 
mit einer Bleikathode und einer Stromdichte $\mathrm{ND}_{100}=12$ Ampère elektrolysiert, so beobachtet man bei normalem Verlauf längere Zeit gar keine Wasserstoffentwickelung; erst allmählich nimmt dieselbe zu und wird schliefslich sehr stürmisch. Die Temperatur steigt bis gegen $55^{\circ}$. Wird die Reaktion nicht zu früh unterbrochen, so gelingt es, fast alles Pyridin zu reduzieren und etwa $95 \%$ der Theorie an Piperidin zu gewinnen. Die Reduktion kann ohne Schaden ohne Diaphragma ausgeführt werden. Auffälligerweise versagte nach längerem Gebrauch der Bleiplatten als Kathoden die Reaktion häufig gänzlich, es trat dann gleich nach Stromschluis heftige Wasserstoffentwickelung und keine oder nur geringe Reduktion ein. Wurde das Pyridin in konz. Schwefelsäure gelöst und im Kathodenraum der Stromwirkung ausgesetzt, so konnte nur Schwefelabscheidung und Schwefelwasserstoffentwickelung, sonst aber keine Reaktion festgestellt werden. Wurde aber die Lösung von Pyridin in konz. Schwefelsäure mit etwas Wasser verdünnt an einer Platinkathode mit niedrigen Stromdichten elektrolysiert, so zeigte sich bald eine Steigerung der Spannung, als deren Ursache sich ein brauner Überzug auf der Kathode herausstellte. Derselbe wurde ab und zu abgestrichen und die Elektrolyse so lange fortgesetzt, bis sich kein Niederschlag mehr anf dem Platinbleche abschied. Diese Substanz wurde ausgewaschen, getrocknet und zerrieben. Sie stellte so ein hellbraunes, amorphes Pulver dar, das in gewöhnlichen Lösungsmitteln, wie Wasser, Alkohol, Äther, Chloroform, Benzol, sowie in Säuren und Alkalien unlöslich war. Es wurde ohne jede Reinigung analysiert. Aus den gefundenen Zahlen konstruierte AHRENs die Formel $\mathrm{C}_{7} \mathrm{H}_{16} \mathrm{~N} \mathrm{SO}_{4}$, ohne freilich zu leugnen, dafs diese des Beweises noch sehr bedürftig, und das Entstehen eines solchen Körpers durch nichts begründet wäre. AHRENs erhielt diesen Körper nur zweimal, spätere Versuche waren in dieser Hinsicht völlig fruchtlos. Er meint, dafs die Bildung dieses Körpers hauptsächlich von der Konzentration der Schwefelsäure abhänge.

Das D.R.-P. 90308 Kl. 12 von E. Merck in Darmstadt (Verfahren zur Darstellung von Piperidin aus Pyridin [und von Dihydrochinolin aus Chinolin]) enthält dieselben Angaben wie die AHRENs'sche Arbeit mit geringfügigen Änderungen, z. B. ist für $\mathrm{ND}_{100}$ hier nicht $12 \mathrm{Amp}$. sondern $10 \mathrm{Amp}$. angegeben.

Um Pyridin als solches in seinen Verbindungen direkt zu bestimmen, sind verschiedene Titrationsmethoden vorgeschlagen worden. 
Die ältere ist von K. E. ScholzE ${ }^{1}$ angegeben worden. Als Indikator dient Eisenchlorid, aus welchem durch Pyridin rotbraunes Eisenoxydhydrat gefällt wird. Mit Normalschwefelsäure wird dann. bis zum völligen Verschwinden des Niederschlages zurücktitriert.

LANG giebt folgende Methode an: Eine abgewogene Menge der pyridinhaltigen Substanz wird in überschüssiger Normalsäure gelöst, Dimethylanilinorange als Indikator zugesetzt und mit $1 / 5^{-}$ norm. Pyridinlösung zurücktitriert. Die weinrote Färbung der sauren Lösung geht beim geringsten Überschufs von Pyridin in goldgelb über. Der Farbenumschlag erschien schärfer, als mit überschüssiger Pyridinlösung versetzt und mit Normal-Saizsäure zurücktitriert wurde. Der Indikator soll in äufserster Verdünnung angewendet werden. Aufser dem oben genannten erwies sich kein Indikator als geeignet.

Es wurde nun, in Anlehnung an Classen und ZaHorskr, versucht, ein Bleichloridpyridin herzustellen. $\mathrm{Zu}$ diesem Zweck wurde siedendes Pyridin mit Bleichlorid versetzt und dann filtriert. Im Filtrat schieden sich nach einigem Stehen weifse Nadeln aus, die abgesaugt und getrocknet wurden. Das Salz war an der Luft sehr zerfliefslich und roch deshalb stark nach Pyridin. Es wurde infolgedessen möglichst schnell analysiert. Zur Pyridinbestimmung wurde das Pyridin direkt mit 1/10-norm. Salzsäure titriert. Als Indikator diente Methylorange. Versuche, die mit Lackmus angestellt wurden, ergaben ein abweichendes und, wie sich herausstellte, falsches Resultat. Das Blei wurde elektrolytisch bestimmt. Das Salz entsprach der Formel $4 \mathrm{PbCl}_{2}, 3 \mathrm{C}_{5} \mathrm{H}_{5} \mathrm{~N}$.

$$
\begin{array}{lcc} 
& \text { Gefunden: } & \text { Berechnet: } \\
\mathrm{Pb} & 61.18 \% & 61.26 \% \\
\mathrm{C}_{5} \mathrm{H}_{5} \mathrm{~N} 17.79 \% & \mathbf{1 7 . 6 2 \%}
\end{array}
$$

Bleinitratpyridin. Kochendes Pyridin wurde mit reinem, festem Bleinitrat versetzt, bis sich nichts mehr löste. Hierbei erwies sich Bleinitrat als ziemlich leicht in Pyridin löslich. Es wurde dann durch ein Heilswasserfilter filtriert. Das Filtrat enthielt reichliche Mengen von Blei und Pyridin und gab bei Zusatz von Wasser einen starken weilsen Niederschlag, der nicht genauer untersucht wurde. Nach kurzem Stehen schied sich aus dem Filtrat ein weilses Salz ab, das abgesaugt und im Exsiccator über Schwefelsäure ge-

1 Ber. deutsch. chem. Ges. 20, 3391. 
trocknet wurde. Es zeigte eine deutliche kleinkrystallinische Struktur. An der Luft war es, wie das vorhergehende Salz, leicht zersetzlich unter Verlust von Pyridin. Es wurde daher möglichst schnell zur Analyse gebracht. Diese ergab die F'ormel $\mathrm{Pb}\left(\mathrm{NO}_{3}\right)_{2} \cdot 2 \mathrm{C}_{5} \mathrm{H}_{5} \mathrm{~N}$.

$\begin{array}{lcc} & \text { Gefunden: } & \text { Berechnet: } \\ \mathrm{Pb} \quad 42.63 \% & 42.42 \% \\ \mathrm{C}_{5} \mathrm{H}_{5} \mathrm{~N} 32.16 \% & 32.38 \%\end{array}$

Bleijodidpyridin wurde hergestellt, indem Bleijodid zu siedendem Pyridin gesetzt wurde, so lange noch etwas in Lösung ging. Die Lösung erfolgte sehr schwierig, durch andauerndes Kochen gelang es, etwas gröfsere Mengen' gelöst zu erhalten. Es wurde vom überschüssigen Bleijodid abfiltriert; im Filtrat schieden sich weifse Kryställchen des Salzes aus, die nicht quantitativ analysiert wurden.

Meine weiteren Versuche bezogen sich nun darauf, Verbindungen von Metallsalzen mit Pyridinsalzen herzustellen.

$\mathrm{Zu}$ diesem $\mathrm{Z}$ wecke wurden als Ausgangsmaterial erst die einfachen, schon von Anderson (s. oben) beschriebenen Pyridinsalze hergestellt.

Pyridinchlorhydrat wurde erhalten, indem Pyridin mit Salzsäure übersättigt und dann abgedampft wurde. Die zurückbleibende krystallinische Masse wurde aus heifsem Alkohol umkrystallisiert. Es bildeten sich weifse Tafeln, die aber an der Luft leicht zerfliefslich waren und darum stets im Exsiccator aufbewahrt wurden.

Zur Herstellung von Pyridinnitrat erwies sich das von ANDERson angegebene Verfahren als nicht praktisch, dieselbe gelang jedoch durch Übersättigen von Pyridin mit gewöhnlicher Salpetersäure und Abdampfen. Das Salz wurde in schön ausgebildeten, bis $10 \mathrm{~cm}$ langen Nadeln erhalten, die dann aus heifsem Alkohol sehr leicht umkrystallisiert wurden. Das Salz ist nicht zersetzlich.

Auch bei der Herstellung des schwefelsauren Pyridins versagte die Vorschrift Andersos's, ein neutrales Gemisch von Pyridin und Schwefelsäure auf dem Wasserbade abzudampfen. Es konnte auf diesem Wege trotz vielfacher Versuche keine krystallinische Masse erhalten werden. Gute Resultate wurden dagegen erzielt, wenn in konz. Schwefelsäure unter ständigem Umrühren immer kleine Mengen von Pyridin eingetragen wurden, bis die ganze Lösung krystallinisch erstarrt war. Es war angebracht, durch Umgeben des Gefäfses mit einer Kältemischung jede gröfsere Erwärmung zu vermindern. Die ausgeschiedene krystallinische Masse wurde aus heifsem Alkohol 
umkrystallisiert; sie war leicht zerfliefslich und mufste deshalb im Exsiccator aufbewahrt werden.

\section{Doppelsalze des Pyridinchlorhydrates.}

Bleichloridpyridinchlorhydrat.

Pyridinchlorhydrat wurde in Wasser gelöst und hierzu Bleichlorid gesetzt, bis sich nichts mehr löste. Es wurde filtriert und stehen gelassen. Es schieden sich bald Krystalle aus, die abgesaugt und getrocknet wurden, zuletzt im Exsiccator über konz. Schwefelsäure. Das trockene, völlig geruchlose Salz repräsentierte feine, glänzende, weilse Nadeln; auch beim längeren Stehenlassen an der Luft trat keine Zersetzung ein. Bei der Analyse wurde Blei elektrolytisch bestimmt; Chlor gewichtsanalytisch und malsanalytisch (Rhodanammonmethode nach VouHand). Zur Bestimmung des Pyridins, das hier an eine Säure gebunden ist, mulste dasselbe erst aus seinem Salze freigemacht werden. $Z u$ diesem $Z$ wecke wurde ein ähnlicher Apparat benutzt, wie er zur Bestimmung des Ammoniaks in Ammonsalzen üblich ist. Durch Kochen mit Kalilauge wird das Pyridin aus seiner Verbindung ausgetrieben; es destilliert über und wird in Vorlagen durch eine abgemessene überschüssige Menge $1 / 10$-norm.-Salzsäure, die mit Methylorange als Indikator versetzt ist, absorbiert. Sobald alles Pyridin übergegangen ist, wird die Reaktion unterbrochen, die überschüssige Normal-Salzsäure mit $1 / 10$-norm.-Kalilauge zurücktitriert, und daraus das Pyridin berechnet. Die Methode gab, wie durch viele Versuche festgestellt wurde, zuverlässige Resultate.

Für das Bleichloridpyridinchlorhydrat wurde die Formel $2 \mathrm{PbCl}_{2} \cdot \mathrm{C}_{5} \mathrm{H}_{5} \mathrm{~N} . \mathrm{HCl}+2 \mathrm{H}_{2} \mathrm{O}$ ermittelt.

$\begin{array}{llc} & \text { Grefunden: } & \text { Berechnet: } \\ \mathrm{Pb} & 58.29 \% & 58.36 \% \\ \mathrm{Cl} & 25.27 \% & 25.14 \% \\ \mathrm{C}_{5} \mathrm{H}_{5} \mathrm{~N} & 11.26,11.28 \% & 11.19 \%\end{array}$

Trotz wiederholter Versuche gelang es nicht, ein Bleichloridpyridinchlorid der Formel $3 \mathrm{PbCl}_{2}, 4 \mathrm{C}_{5} \mathrm{H}_{5} \mathrm{~N} . \mathrm{HCl}$ (s. oben Classen und $\mathrm{Z}_{\mathrm{AHORSKY}} \mathrm{zu}$ erhalten.

Eisenchloridpyridinchlorhydrat.

Eine Lösung von Pyridinchlorhydrat wurde mit überschüssigem festen Eisenchlorid versetzt, vom nicht gelösten abfiltriert und stehen 
gelassen. Es schieden sich bald Krystalle aus, die mit kaltem Wasser ausgewaschen, abgesaugt, zwischen Flielspapier abgeprelst und endlich im Exsiccator über konz. Schwefelsäure getrocknet wurden. Im trockenen Zustande zeigte das Salz schöne, seidenglänzende, gelbbraune Nadeln, die sich unter dem Mikroskop als Aggregate kleiner Kryställchen erwiesen. Es zersetzte sich an der Luft ebenso wenig wie die vorherbeschriebene Verbindung. Das $\mathrm{Salz}$ entsprach der Formel $\mathrm{Fe}_{2} \mathrm{Cl}_{6} \cdot 3 \mathrm{C}_{5} \mathrm{H}_{5} \mathrm{~N} . \mathrm{HCl} .3 \mathrm{H}_{2} \mathrm{O}$.

Die Analysen, bei denen Eisen als Oxydhydrat, die anderen Bestandteilen nach den oben beschriebenen Methoden bestimmt wurden, ergaben:

$\begin{array}{lcc} & \text { Gefunden: } & \text { Berechnet: } \\ \mathrm{Fe} & \mathbf{1 5 . 4 2 , 1 5 . 4 3 \%} & 15.44 \% \\ \mathrm{Cl} & 44.17 \% & 44.01 \% \\ \mathrm{C}_{5} \mathrm{H}_{5} \mathrm{~N} & \mathbf{3 2 . 4 4} " & 32.64 \%\end{array}$

Baryumchloridpyridinchlorhydrat.

Die wässerige Lösung von Pyridinchlorhydrat wurde mit Baryumchlorid versetzt und filtriert. Im Filtrat schieden sich weifse Krystalle aus, die abgesaugt und getrocknet wurden. Die gut ausgebildeten Krystalle erwiesen sich als luftbeständig; sie waren glänzend, farblos und durchscheinend. Das Salz löste sich sehr leicht in heifsem, schwerer in kaltem Wasser; auch in verdünnten Mineralsäuren war es leicht löslich. Durch Analyse wurde für das Salz die Formel $\left(\mathrm{BaCl}_{2}\right)_{3} \cdot \mathrm{C}_{5} \mathrm{H}_{5} \mathrm{~N}$.HCl. $\mathrm{H}_{2} \mathrm{O}$ ermittelt.

$\begin{array}{lcc} & \text { Gefunden: } & \text { Berechnet: } \\ \mathrm{Ba} & 54.05,54.17 \% & 54.29 \% \\ \mathrm{Cl} & 32.54 \% & 32.76 \% \\ \mathrm{C}_{5} \mathrm{H}_{5} \mathrm{~N} & 10.28 \% & 10.44 \%\end{array}$

Magnesiumchloridpyridinchlorhydrat

wurde auf gleiche Weise hergestellt. Das Salz war aufserordentlich voluminös und zerfliefslich. Beim Stehen an der Luft zerflols die ursprünglich gut charakterisierte krystallinische Masse vollständig, wobei Pyridin entwich. Die Bestandteile des Salzes wurden infolge der grofsen Unbeständigkeit nur qualitativ festgestellt.

Silberchloridpyridinchlorhydrat, weifse, kleinkrystallinische Masse. 
Aus Pyridinchlorhydrat mit Manganchlorür $\left(\mathrm{MnCl}_{2}\right)$ konnten zwei Salze dargestellt werden.

\section{Manganchlorürdipyridinchlorhydrat}

wurde erhalten, indem die wässerige Lösung von Pyridinchlorhydrat nit reinem Manganchlorür versetzt wurde, und dann, wie oben beschrieben, vom ungelösten abfiltriert, und die im Filtrat ausgeschiedenen Krystalle abgesaugt und getrocknet wurden. Das Salz war gelb gefärbt. Es löste sich leicht in Wasser und Alkohol, verlor aber hierbei seine Färbung, die in rosa überging. In $\ddot{A}$ ther war es unlöslich. Es gelang nicht, das Salz unverändert umzukrystallisieren. Sein Schmelzpunkt liegt bei $170-175^{\circ}$. Dem Salz ist die Formel $\mathrm{MnCl}_{2} \cdot 2 \mathrm{C}_{5} \mathrm{H}_{5} \mathrm{~N}$. $\mathrm{HCl}$ zuzuschreiben.

$\begin{array}{lrc} & \text { Gefunden: } & \text { Berechnet: } \\ \mathrm{Mn} & 15.53 \% & 15.41 \% \\ \mathrm{C}_{5} \mathrm{H}_{5} \mathrm{~N} \quad 43.95 \% & 44.25 \%\end{array}$

Manganchlorürpyridinchlorhydrat.

Manganchlorür $\left(\mathrm{MnCl}_{2}\right)$ und Pyridinchlorhydrat wurden im Verhältnis ihrer Molekulargewichte in Wasser gelöst und die Lösung auf dem Wasserbade abgedampft. Der feste Körper, welcher hierbei zurückblieb, wurde getrocknet, in Alkohol gelöst und filtriert. Im Filtrat schieden sich bald Krystalle aus, die abgesaugt und getrocknet wurden. Das mattrosa gefärbte Salz zersetzte sich, wie auch das vorige, beim Stehen an der Luft nicht. Es konnte, entgegen dem oben beschriebenen, aus Wasser und Alkohol unverändert umkrystallisiert werden. Durch die Analyse wurde dafür die Formel $\mathrm{MnCl}_{2} \cdot \mathrm{C}_{5} \mathrm{H}_{5} \mathrm{~N}$.HCl festgestellt.

$$
\begin{array}{lcc} 
& \text { Gefunden: } & \text { Berechnet: } \\
\mathrm{Mn} & 22.65 \% & 22.73 \% \\
\mathrm{C}_{5} \mathrm{H}_{5} \mathrm{~N} 32.48 \% & 32.64 \%
\end{array}
$$

\section{Doppelsalze des Pyridinnitrates.}

Silbernitratpyridinnitrat.

$\mathrm{Zu}$ seiner Darstellung wurde eine Lösung von Pyridinnitrat in Wasser mit Silbernitrat versetzt, auf dem Wasserbade abgedampft, und der zurückbleibende feste Körper in heif́sem Wasser gelöst. Es wurde filtriert, die sich aus dem Filtrat leicht ausscheidenden Krystalle abgesaugt und getrocknet; endlich wurde das Salz aus 
heilsem Alkohol umkrystallisiert. Es war leicht löslich in Wasser und Alkohol, unlöslich in Äther. Das Salz bildete schön ausgeprägte, seidenglänzende Nadeln, zum Teil von sehr beträchtlicher Länge. Unter dem Mikroskop konnte man häufig garbenförmige Zusammenlagerungen beobachten. Das Salz war an der Luft nicht zersetzlich. Wurde es in einem Porzellantiegel über dem Bunsenbrenner erhitzt, so fand leicht Reduktion statt; es erfolgte die Bildung eines deutlichen Silberspiegels.

Das Salz entsprach der Formel $5 \mathrm{AgNO}_{3} \cdot 3 \mathrm{C}_{5} \mathrm{H}_{5} \mathrm{~N} \cdot \mathrm{HNO}_{3}$.

$\begin{array}{lcc}\quad & \text { Gefunden: } & \text { Berechnet: } \\ \mathrm{Ag} & \mathbf{4 2 . 1 6 \%} \% & 42.32 \% \\ \mathrm{C}_{5} \mathrm{H}_{5} \mathrm{~N} & 18.72 \% & 18.57 \%\end{array}$

Uranylnitratpyridinnitrat

wurde durch Abdampfen einer Lösung der beiden Komponenten auf dem Wasserbade als fester, amorpher Körper gewonnen, der dann in heifsem Wasser gelöst wurde und daraus beim Stehenlassen leicht auskrystallisierte. Die abgesaugten und getrockneten Krystalle wurden noch aus heifsem Alkohol umkrystallisiert. Sie zeigten gelbe Färbung, waren gut ausgebildet und zersetzten sich beim Stehen an der Luft nicht. Das Salz löste sich leicht in Wasser und Alkohol, dagegen war es in Äther unlöslich. Unter dem Mikroskop sah man hübsch ausgebildete Nadeln, die federförmig angeordnet waren. Das Salz entsprach der Formel: $\mathrm{UrO}_{2}\left(\mathrm{NO}_{3}\right)_{2} \cdot 3 \mathrm{C}_{5} \mathrm{H}_{5} \mathrm{~N} \cdot \mathrm{HNO}_{3} \cdot 2 \mathrm{H}_{2} \mathrm{O}$ gemäls den Analysenresultaten:

$\begin{array}{lcc} & \text { Gefunden: } & \text { Berechnet: } \\ \mathrm{Ur} & \mathbf{2 7 . 8 3} \% & \mathbf{2 7 . 9 7} \% \\ \mathrm{C}_{5} \mathrm{H}_{5} \mathrm{~N} 27.48 \% & 27.62 \%\end{array}$

Kupfernitratpyridinnitrat

wurde genau wie das vorhergehende Salz dargestellt und ebenfalls aus heifsem Alkohol umkrystallisiert. Die blauen Krystalle waren gut ausgebildet. Sie waren löslich in Wasser und Alkohol, unlöslich in Äther. An der Luft zeigte das Salz sich beständig. Bei mikroskopischer Untersuchung sah man lange prismatische Nadeln, die besonders häufig garbenförmige Anordnung zeigten. Das Salz wurde nur qualitativ untersucht.

Zur Herstellung des

Bleinitratpyridinnitrat

wurde die wässerige Lösung des Pyridinnitrates mit festem Blei- 
chlorid in reichlicher Menge versetzt, sodann vom ungelösten abfiltriert und stehen gelassen. Die ausgeschiedenen Krystalle wurden nach der üblichen Methode behandelt. Die gelben Krystalle waren an der Luft ziemlich leicht zersetzlich und rochen deshalb nach Pyridin. Für das Salz wurde die Formel: $\mathrm{Pb}\left(\mathrm{NO}_{3}\right)_{2} \mathrm{C}_{5} \mathrm{H}_{5} \mathrm{~N} \cdot \mathrm{HNO}_{3}$ festgestellt.

$\begin{array}{lcc} & \text { Gefunden: } & \text { Berechnet: } \\ \mathrm{Pb} & 43.51 \% & 43.64 \% \\ \mathrm{C}_{5} \mathrm{H}_{5} \mathrm{~N} \quad 16.87 \% & 16.75 \%\end{array}$

Bei längerem Stehen an der Luft ging Pyridin fort; zurück scheint schlielslich einfaches Bleinitrat $\left(\mathrm{PbN}_{2} \mathrm{O}_{6}\right) \mathrm{zu}$ bleiben. Dieses Resultat ergiebt sich aus mehreren ausgeführten Bleibestimmungen des Salzes, das längere Zeit frei an der Luft gelegen hatte. Die Ergebnisse waren:

Gefunden:

\section{I.}

$\mathrm{Pb} \quad 59.48 \%$
U.

$60.92 \%$
Berechnet

für $\mathrm{Pb}\left(\mathrm{NO}_{3}\right)_{2}$ :

$62.42 \%$

Die erste Analyse wurde früher ausgeführt. Es ist leicht ersichtlich, dals der Bleigehalt einer Grenze, nämlich dem Bleigehalt des einfachen Nitrates zustrebt. Die letzten Reste Pyridin scheinen schwierig aus dem Salze heraus zu gehen, da die Zersetzung, welche anfangs sehr schnell erfolgte, später so langsam weiterging, dass in der That reines Bleinitrat nicht erhalten wurde.

\section{Kobaltnitratpyridinnitrat}

wurde hergestellt, indem ungefähr 2 Teile Kobaltnitrat mit 3 Teilen Pyridinnitrat in Wasser gelöst, abgedampft, der feste Rückstand mit heifsem Wasser aufgenommen, filtriert und stehen gelassen wurde. Es schieden sich im Filtrat schöne rote Krystalle aus, die abgesaugt und getrocknet wurden. Das Salz erwies sich als beständig; auch bei längerem Liegen an der Luft wurde es nicht zersetzt. Formel: $\mathrm{Co}\left(\mathrm{NO}_{3}\right)_{2} \cdot 3 \mathrm{C}_{5} \mathrm{H}_{5} \mathrm{~N} \cdot \mathrm{HNO}_{3}$.

$\begin{array}{lrc} & \text { Gefunden: } & \text { Berechnet: } \\ \text { Co } & 9.65 \% & 9.54 \% \\ \mathrm{C}_{5} \mathrm{H}_{5} \mathrm{~N} & 39.07 \% & 38.98 \%\end{array}$

Strontiumnitratpyridinnitrat

wurde leicht aus Strontiumnitrat und Pyridinnitrat erhalten. Es bildete ein weilses, in gut ausgebildeten Formen krystallisierendes 
Salz, das völlig geruchlos war, sich auch an der Luft nicht zersetzte. Es entsprach der Formel: $\mathrm{Sr}\left(\mathrm{NO}_{3}\right)_{2} \cdot \mathrm{C}_{5} \mathrm{H}_{5} \mathrm{~N} \cdot \mathrm{HNO}_{3}$.

$$
\begin{array}{lcc} 
& \text { Gefunden: } & \text { Berechnet: } \\
\mathrm{Sr} & 24.66 \% & 24.75 \% \\
\mathrm{C}_{5} \mathrm{H}_{5} \mathrm{~N} & 22.50 \% & 22.35 \%
\end{array}
$$

\section{Doppelsalze des Pyridinsulfates.}

Nickelsulfatpyridinsulfat.

Nickelsulfat und Pyridinsulfat wurden in Wasser gelöst und die Lösung auf dem Wasserbad zur Trockne eingedampft. Der Rückstand wurde in heifsem Wasser aufgenommen und filtriert. Im Filtrat schieden sich grüne Krystalle aus, die abgesaugt, zwischen Fliefspapier abgeprelst und endlich im Exsiccator über konz. Schwefelsäure getrocknet wurden. Das Salz war vollkommen geruchlos; beim Stehen an der Luft konnte keine Zersetzung durch Pyridinabgang wahrgenommen werden, doch verwitterte das Salz leicht an der Oberfläche, indem es einen Teil seines Krystallwassers verlor. Formel: $3 \mathrm{NiSO}_{4} \cdot 2 \mathrm{C}_{5} \mathrm{H}_{5} \mathrm{~N} \cdot \mathrm{H}_{2} \mathrm{SO}_{4} \cdot 10 \mathrm{H}_{2} \mathrm{O}$.

$$
\begin{array}{lcc} 
& \text { Gefunden: } & \text { Berechnet: } \\
\mathrm{Ni} \text { (Mittel) } & \mathbf{1 7 . 5 8 \%} & 17.47 \% \\
\mathrm{C}_{5} \mathrm{H}_{5} \mathrm{~N} & 15.71 \% & 15.86 \%
\end{array}
$$

Nickel wurde sowohl gewichtsanalytisch wie auch elektrolytisch aus ammoniakalischer Lösung bestimmt.

\section{Kobaltsulfatpyridinsulfat}

wurde auf analoge Weise wie das vorhergehende Salz erhalten. Es bildete einen roten, mikrokrystallinischen Körper ohne ausgeprägten Charakter. Es war, wie das obige, völlig geruchlos und liefs beim Stehen an der Luft kein Pyridin fortgehen. Formel: $4 \mathrm{CoSO}_{4} \cdot \mathrm{C}_{5} \mathrm{H}_{5} \mathrm{~N}$. $\mathrm{H}_{2} \mathrm{SO}_{4} \cdot 10 \mathrm{H}_{2} \mathrm{O}$.

$\begin{array}{lcc} & \text { Gefunden: } & \text { Berechnet: } \\ \text { Co } & 23.63 \% & 23.84 \% \\ \mathrm{SO}_{4} & 49.24 \% & 49.35 \% \\ \mathrm{C}_{5} \mathrm{H}_{5} \mathrm{~N} & 8.20 \% & 8.12 \%\end{array}$

Auch hier wurde das Kobalt elektrolytisch aus ammoniakalischer Lösung unter Zusatz von schwefelsaurem Ammon abgeschieden.

Es wurde versucht, Doppelsalze des Bleisulfates und des Baryumsulfates zu erhalten. Es war dies aber nicht möglich. 


\section{Zinksulfatpyridinsulfat.}

Es wurden zu diesem Zwecke die Komponenten in Wasser gelöst und auf dem Wasserbade abgedampft. Aus der filtrierten Lösung des so gebildeten festen Körpers schieden sich weilse Krystalle aus, die abgesaugt und getrocknet wurden. Die zuerst durchsichtigen, glänzenden Krystalle verwitterten leicht beim Liegen an der Luft; sie wurden undurchsichtig und glanzlos. Pyridin ging jedoch hierbei nicht fort. Durch eine an auserlesenen, frischen Krystallen ausgeführte Analyse wurde für das Salz die Formel: $3 \mathrm{ZnSO}_{4} \cdot 2 \mathrm{C}_{5} \mathrm{H}_{5} \mathrm{~N} \cdot \mathrm{H}_{2} \mathrm{SO}_{4}, 2 \mathrm{H}_{2} \mathrm{O}$ ermittelt.

$\begin{array}{lcc} & \text { Gefunden: } & \text { Berechnet: } \\ \mathrm{Zn} & 15.72 \% & 15.87 \% \\ \mathrm{SO}_{4} & 40.47 \% & 40.29 \% \\ \mathrm{C}_{5} \mathrm{H}_{5} \mathrm{~N} & 13.20 \% & 13.27 \%\end{array}$

Eisenoxydulsulfatpyridinsulfat.

Reines Eisensulfat und Pyridinsulfat wurden in viel heifsem Wasser gelöst, ein Teil des Wassers abgedampft und dann filtriert. Es schieden sich im Filtrat langsam durchsichtige weilse Krystalle aus, die ähnlich dem einfachen Eisensalz krystallisierten. Diese wurden abgesaugt, zwischen Filtrierpapier abgeprefst und getrocknet. An der Luft veränderte sich das Salz sehr leicht; es fing an, an der Oberfläche zu verwittern und nahm einen fast körnigen Habitus an. In Alkohol und Wasser war es leicht löslich.

Formel: $8 \mathrm{FeSO}_{4} \cdot \mathrm{C}_{5} \mathrm{H}_{5} \mathrm{~N} \cdot \mathrm{H}_{2} \mathrm{SO}_{4}+3 \mathrm{H}_{2} \mathrm{O}$.

$\begin{array}{lcc} & \text { Gefunden: } & \text { Berechnet: } \\ \mathrm{Fe} & 23.39 \% & 23.18 \% \\ \mathrm{SO}_{4} & 44.49 \% & 44.72 " \\ \mathrm{C}_{5} \mathrm{H}_{5} \mathrm{~N} & 4.04 \% & 4.09 \%\end{array}$

Doch dürfte es, infolge der sehr komplizierten Formel, dahingestellt bleiben, ob man es hier mit einem einheitlichen Körper zu thun hat.

\section{Aluminiumsulfatpyridinsulfat}

wurde auf ähnliche Weise wie die vorhergehenden Verbindungen hergestellt. Es bildete eine weilse, glanzlose, mikrokrystallinische Masse, die schon bei längerem Liegen an ter Luft zerflols, bedeutend schneller bei etwas erhöhter Temperatur zu einer klebrigen Masse, die stark nach Pyridin roch. Es war leicht löslich in Wasser 
und Alkohol und wurde auch aus letzterem unverändert umkrystallisiert.

Das Salz entsprach der Formel: $\mathrm{Al}_{2}\left(\mathrm{SO}_{4}\right)_{3} \cdot 4 \mathrm{C}_{5} \mathrm{H}_{5} \mathrm{~N} \cdot \mathrm{H}_{2} \mathrm{SO}_{4}+6 \mathrm{H}_{2} \mathrm{O}$.

$\begin{array}{lrc} & \text { Gefunden: } & \text { Berechnet: } \\ \mathrm{Al} & 4.73 \% & 4.65 \% \\ \mathrm{C}_{5} \mathrm{H}_{5} \mathrm{~N} 27.20 \% & 27.29 \%\end{array}$

Kupfersulfatpyridinsulfat

Es wurden zur Synthese die Mengen der Komponenten im Verhältnis der Molekulargewichte gewählt. Das Salz bildete blaue, gut ausgebildete, dem einfachen Kupfersulfat ähnliche Krystalle, die in Wasser sehr leicht löslich waren, und deshalb nur schwierig, nachdem man sie mehrere Tage hatte stehen lassen, sich aus der Lösung ausschieden. Aus Alkohol konnten sie gut umkrystallisiert werden. Das Salz war vollständig geruchlos und zerflofs an der luft nicht. Dagegen fing es bei längerem Liegen an der Luft an, durch Krystallwasserabgang etwas zu verwittern. Es bildete sich eine weifse Schicht an der Oberfläche, wie bei dem einfachen Kupfersalz. Es dürfte daher wohl auch das Doppelsalz in wasserfreiem Zustande farblos sein.

Das Salz hatte die Formel: $3 \mathrm{CuSO}_{4} \cdot \mathrm{C}_{5} \mathrm{H}_{5} \mathrm{~N}_{2} \cdot \mathrm{H}_{2} \mathrm{SO}_{4} \cdot 18 \mathrm{H}_{2} \mathrm{O}$.

$\begin{array}{lcc} & \text { Gefunden: } & \text { Berechnet: } \\ \mathrm{Cu} & 19.63 \% & 19.33 \% \\ \mathrm{SO}_{4} & 39.47 " & 39.28 \% \\ \mathrm{C}_{5} \mathrm{H}_{5} \mathrm{~N} & 8.20 " & 8.08 \%\end{array}$

Im allgemeinen hat man also in den Metalldoppelsalzen des Pyridinchlorhydrates, des Pyridinnitrates und des Pyridinsulfates gut charakterisierte krystallinische Verbindungen vor sich. Die meisten derselben sind an der Luft durchaus beständig, nur einige verwittern oder zerfliefsen und geben dabei auch Pyridin ab. Zum grölsten Teil sind diese Körper daher auch geruchlos.

Die zuerst beschriebenen Verbindungen dagegen, in denen einfach Pyridin an ein Metallsalz angelagert ist, sind recht unbeständig. Sie verlieren leicht ihr Pyridin oder einen grossen Teil davon und riechen daher ziemlich stark.

Für diese letzteren Salze wurde auch eine neue Herstellungsmethode ausgeführt. Es stellte sich heraus, dals in den wässerigen Lösungen der einfachen Pyridinsalze (Pyridinchlorhydrat etc.) Metall- 
oxyde zum Teil leicht löslich sind. Um einige Beispiele anzuführen, gelang es, das schon beschriebene

\section{Bleichloridpyridin}

auf folgende Weise darzustellen. In die wässerige Lösung des Pyridinchlorhydrates wurde reichlich Bleioxyd eingetragen, das sich ziemlich leicht löste. Es wurde abfiltriert; im Filtrat waren grofse Mengen Pyridin und Blei nachzuweisen. Beim Stehenlassen schieden sich Krystalle des oben beschriebenen Salzes aus.

Ebenso konnte das

\section{Bleinitratpyridin}

durch Lösen von Bleioxyd in Pyridinnitrat leicht erhalten werden. Dagegen gelang auf demselben Wege die Synthese des Bleisulfatpyridins nicht.

Zum Schlusse der rein chemischen Untersuchungen wurde noch versucht, einige der noch nicht dargestellten einfachen Pyridinsalze zu erhalten.

$$
\text { Pyridinphosphat. }
$$

Reine Phosphorsäure von ungefähr Syrupkonsistenz wurde bis zur neutralen Reaktion mit Pyridin versetzt und auf dem Wasserbade eingedampft, bis sich ein dicker schwerbeweglicher Syrup gebildet hatte. Trotz aller Versuche gelang es im Anfang nicht, einen festen Körper zu erhalten; erst nach sehr langem Stehen erstarrte er zu einer amorphen Masse, die sich aber als ziemlich beständig erwies, an der Luft nur in geringem Malse zerflofs und nicht nach Pyridin roch. Es gelang nicht, durch Umkrystallisieren die Verbindung krystallinisch zu erhalten.

Das Salz entsprach der Formel: $2 \mathrm{H}_{3} \mathrm{PO}_{4} \cdot \mathrm{C}_{5} \mathrm{H}_{5} \mathrm{~N} \cdot \mathrm{H}_{2} \mathrm{O}$.

$$
\begin{array}{rc}
\text { Gefunden: } & \text { Berechnet: } \\
\mathrm{H}_{3} \mathrm{PO}_{4} 66.66 \% & 66.89 \% \\
\mathrm{C}_{5} \mathrm{H}_{5} \mathrm{~N} 26.87 \% & 26.96 \%
\end{array}
$$

Um das

$$
\text { Pyridinwolframat }
$$

darzustellen, wurden berechnete Mengen von Pyridinsulfat und Natriumwolframat in Wasser gelöst. Beim Zusammenbringen der beiden klaren Lösungen bildete sich ein starker weifser Niederschlag, der auf der Saugpumpe abfiltriert und mit ca. 50\% kaltem Alkohol ausgewaschen wurde. Er war unlöslich in Alkohol, leicht löslich 
in heifsem, schwerer in kaltem Wasser. Aus heifsem Wasser konnte er umkrystallisiert werden, behielt jedoch hierbei denselben mikrokrystallinischen Habitus.

Formel: $2 \mathrm{WO}_{2}(\mathrm{OH})_{2} \cdot \mathrm{C}_{5} \mathrm{H}_{5} \mathrm{~N}$.

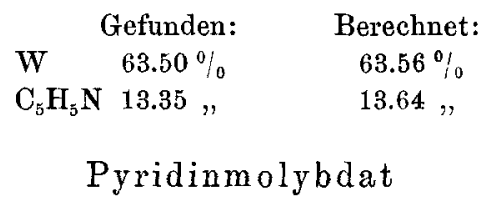

endlich wurde hergestellt, indem Molybdänsäure mit Natronlange versetzt wurde, die Lösung des so gebildeten Natriummolybdates filtriert und mit einer filtrierten Lösung von Pyridinsulfat oder Pyridinacetat versetzt wurde. Der weifse Niederschlag, welcher hierbei ausfiel, wurde abgesaugt und mit verdünntem Alkohol nachgewaschen. Das Salz war in heifsem Wasser leicht, in kaltem schwer löslich.

$$
\begin{array}{lc}
\text { Formel : } 3 \mathrm{MoO}_{2}(\mathrm{OH})_{2} \cdot \mathrm{C}_{5} \mathrm{H}_{5} \mathrm{~N} . \\
\text { Gefunden: } & \text { Berechnet: } \\
\mathrm{Mo}^{51.17 \%} & 50.97 \% \\
\mathrm{C}_{5} \mathrm{H}_{5} \mathrm{~N} \quad 14.07 \% & 13.98 \%
\end{array}
$$

Elektrochemischer Teil. Ich beabsichtigte, nach Wiederholung der AHRENs'schen Versuche auf denselben weiterzubauen; durch die abweichenden Ergebnisse aber, die ich erhielt, wurde ich gezwungen, mich mit diesen Versuchen eingehender zu beschäftigen.

Als Zersetzungsgefäls benutzte ich ein kleines, rundes Elementenglas, als Diaphragma eine runde Thonzelle. Die Elektroden wurden bei den ersten Versuchen aus Blei gewählt, die Anode von der Höhe der Flüssigkeitsschicht umgab rings die Thonzelle; in dieser hatte die Kathode, ein Bleiblech von ca. $34 \mathrm{qcm}$ Oberfläche, Platz gefunden. In der Thonzelle befand sich eine Lösung von 1 Teil Pyridin in 10 Teilen $10 \%$ iger Schwefelsäure, um die Zelle herum eine $10 \%$ ige Schwefelsäurelösung, genau den AnRENs'schen Angaben entsprechend.

Versuch I. $\mathrm{ND}_{100}=12 \mathrm{Amp}$. Die Pyridinsulfatlösung im Kathodenraum zeigte anfangs eine Temperatur von $20^{\circ} \mathrm{C}$. Gleich zu Beginn der Elektrolyse zeigte sich lebhafte Wasserstoffentwickelung, die sehr bald zunahm. Die Temperatur im Kathodenraum stieg dabei zuerst langsam auf $30^{\circ}$, dann sehr schnell bis $80^{\circ}$ und 
wieder langsam bis fast $100^{\circ}$, wo sie dann konstant bis zum Ende der Elektrolyse blieb.

Die Stromstärke $(J)$, die zuerst 4 Amp. betrug, nahm langsam ab. Sobald die Temperatur stieg, zeigten sich auch starke Schwankungen des Ampèremeters, die sich regelmälsig in kurzen Zwischenräumen wiederholten. Indessen nahm die Flüssigkeit in der Thonzelle, wohl hauptsächlich durch Verdunstung infolge der starken Wärmeentwickelung, langsam ab. Nach ca. $3^{1} / 2$ Stunden war $J$ auf 0.3 Amp. gefallen. Es wurde nun ausgeschaltet. An der Kathode befand sich ein dichter brauner Überzug, der wohl mit dem von AHrens beschriebenen identisch war. Er erwies sich als unlöslich in allen Lösungsmitteln und zeigte durchaus kein charakterisiertes Aussehen, liels sich aber leicht trocknen und zu einem hellbraunen Pulver verreiben. Dieser Körper dürfte wohl als Verharzung aufgefafst werden. In der geringen, im Kathodenraum zurückgebliebenen Flüssigkeit konnte kein Piperidin nachgewiesen werden.

Versuch II. Die Bedingungen waren genau wie bei I gewählt. $\mathrm{ND}_{100}=12 \mathrm{Amp}$. Es wurden in kurzen Zwischenräumen Beobachtungen angestellt, und zwar von der Temperatur im Kathodenraume $(t)$ und der Stromstärke $(J)$. Die erhaltenen Resultate waren folgende:

Anfang $12^{20}$.

\begin{tabular}{c|l|l|l|l|l}
\hline \hline Zeit & \multicolumn{1}{|c|}{$t$} & $J$ & Zeit & \multicolumn{1}{|c|}{$t$} & $J$ \\
\hline \hline $12^{30}$ & $36.5^{0}$ & 4.58 & $1^{18}$ & $97^{0}$ & 3.22 \\
$12^{36}$ & $39^{0}$ & 4.58 & $1^{23}$ & $98^{0}$ & 3.2 \\
$12^{48}$ & $43.2^{0}$ & 4.56 & $1^{26}$ & $98.5^{0}$ & 3.1 \\
$12^{58}$ & $46.5^{0}$ & 4.56 & $1^{33}$ & $99^{0}$ & 3.0 \\
$1^{3}$ & $54^{0}$ & 4.25 & $1^{35}$ & $99.3^{0}$ & 2.8 \\
$1^{8}$ & $75^{0}$ & 3.6 & $1^{37}$ & $99.3^{0}$ & 2.7 \\
$1^{13}$ & $89.5^{0}$ & 3.4 & & &
\end{tabular}

Hier wurde ausgeschaltet. An der Kathode zeigte sich wieder der erwähnte braune Körper; die Flüssigkeit war hellbraun gefärbt. Piperidin war nur in sehr geringen Mengen neben Pyridin nachzuweisen. Während der Elektrolyse nahm die Wasserstoffentwickelung konstant zu und wurde schliefslich sehr heftig.

Versuch III. Die IJösungen erhielten dieselbe Konzentration, wie bei I und II. Um sich möglichst weit von den Bedingungen zu entfernen, die Ahrens als Ursache falscher Resultate angiebt, wurde die Stromdichte auf das doppelte, d. h. 24 Amp. erhöht. 
Der Versuch ergab im wesentlichen dasselbe Resultat wie die früheren. Es trat sofort nach Stromschlufs Wasserstoffentwickelung auf, die bald stärker und endlich sehr heftig wurde. Die Temperatur stieg in 25 Minuten von $20^{\circ}$ auf $79^{\circ}$, die Stromstärke nahm ständig ab. Als Resultat zeigte sich an der Kathode wieder der braune Überzug, der auch früher erhalten worden war. In der schwach gefärbten Kathodenflüssigkeit war neben grofsen Mengen unveränderten Pyridins nur wenig Piperidin nachzuweisen, wie sowohl durch den Geruch, wie auch durch die sehr empfindliche Reaktion mit Benzoylchlorid - es bildet sich festes Benzoylpiperidin, während mit Pyridin keine Verbindung entsteht - nachgewiesen wurde.

Versuch IV. AHRENs giebt an, dafs auch zu starke Konzentration der Schwefelsäure ein Hinderungsgrund für den glatten Verlauf der Reaktion sei. Es wurde deshalb hier eine nur $6 \%$ ige Schwefelsäure angewendet. $\mathrm{ND}_{100}$ war $=20 \mathrm{Amp}$.

Anfang $4^{25}$.

\begin{tabular}{c|c|c}
\hline \hline Zeit & $t$ & $J$ \\
\hline \hline $4^{30}$ & $40^{0}$ & 7.1 \\
$4^{33}$ & $75^{0}$ & 7.4 \\
$4^{36}$ & $88^{0}$ & 7.6 \\
$4^{39}$ & $95^{0}$ & 6.4 \\
$4^{44}$ & $99^{0}$ & 4.4 \\
$4^{48}$ & $100^{0}$ & 2.4
\end{tabular}

Die Wasserstoffentwickelung wurde aufserordentlich heftig. Ein Ampèremeter, das zur Messung des Polarisationsstromes nach Unterbrechung des Hauptstromes an der Zersetzungszelle angeschaltet war, zeigte zuerst 3.5 Amp., fiel aber bald auf 0.4 Amp. Zur Messung der Spannung des Polarisationsstromes wurde nach Unterbrechung des Hauptstromes ein Voltmeter angeschaltet; dieses zeigte ca. 1.2 Volt. Das Endresultat war das gleiche wie bei den vorhergehenden Versuchen: auch hier wurde der beschriebene braune Körper erhalten.

Versuch V. Zu Elektroden wurde hier Kohle verwendet. Die Kathode war ein Stab von $34 \mathrm{qcm}$ Oberfläche, als Anode dienten zwei Platten, die zu Seiten der Thonzelle angebracht waren. Die Schwefelsäure wurde wieder $10 \%$ ig verwendet, da sich durch schwächere Konzentration kein Vorteil ergeben hatte. Auch wurde 
wieder mit der Stromdichte $\mathrm{ND}_{100}=12 \mathrm{Amp}$. gearbeitet. Die Elektrolyse verlief folgendermalsen:

\begin{tabular}{c|c|c}
\hline Zeit & $t$ & $J$ \\
\hline \hline $12^{18}$ & $\mathbf{2 4 ^ { 0 }}$ & 4.3 \\
$12^{84}$ & $\mathbf{4 4}^{0}$ & 4.35 \\
$12^{40}$ & $46^{0}$ & 4.35 \\
$12^{50}$ & $50^{0}$ & 4.37 \\
1 & $53^{0}$ & 4.4 \\
$1^{\mathbf{3 5}}$ & $100^{0}$ & 3.4 \\
$1^{55}$ & $100^{0}$ & 3.2
\end{tabular}

Tine Probe des braunen Rückstandes, der sich auch hier an der Kathode gebildet hatte, wurde mit Natronlauge stark gekocht, um eine event. darin befindliche Base abzuscheiden. Dann wurde mit Äther ausgeschüttelt. Beim Verdunsten auf dem Uhrglase blieb ein weifser Körper zurück, der in Essigsäure leicht gelöst wurde und so mit Molybdänsäure einen Niederschlag gab. Die Hauptmenge der braunen Substanz wurde mit Natronlauge stark gekocht: es schied sich ein kleinkrystallinischer Körper ab. Es wurde noch einmal erhitzt und filtriert; im Filtrat krystallisierte der Körper wieder aus. Ein Versuch, ihn im Fraktionierkölbchen mit Wasserdämpfen überzutreiben, gelang nicht; es destillierte nur eine gelblich bräunliche Flüssigkeit. Leider gelang es nicht, den weilsen Körper, der nur in sehr geringer Menge erhalten worden war, zu analysieren: er wurde beim Filtrieren vom Papier aufgenommen, und es war nicht möglich, ihn wieder herauszubekommen.

Spätere Versuche, den Körper wieder darzustellen, verliefen ganz fruchtlos.

Versuch IV. Die Anordnung des Versuches wurde im wesentlichen beibehalten. Auch die Konzentration der Schwefelsäure war wieder zu $10 \%$ genommen worden. Als Kathode diente dieses Mal ein Platinblech; das Arbeiten mit einem solchen bietet besonders in betreff auf Sauberkeit grolse Annehmlichkeiten; auch ist nicht einzusehen, weshalb es hindernd auf die Bildung von Piperidin wirken könnte. Es wurde mit sehr hohen Stromdichten (25-30 Amp.) gearbeitet. Demgemäls war die Reaktion auch sehr heftig; sofort nach Stromschlufs trat heftige Wasserstoffentwickelung ein, und in 
kurzer Zeit bildete sich der braune Überzug an der Kathode. In ungefähr 5 Minuten sank die Stromstärke, die zuerst = $10 \mathrm{Amp}$. genommen worden war, auf 2-3 Amp. Es wurde dann ausgeschaltet, der reichhaltig gebildete braune Überzug von der Kathode abgestrichen, und der Prozels begann aufs neue, indem das Ampèremeter nach Einschaltung sofort wieder auf die frühere Höhe stieg, um in kurzer Zeit, infolge des neu gebildeten Überzuges auf 2 Amp. zu sinken. So wurde der Prozefs häufig wiederholt, und es gelang infolge dessen, eine stattliche Quantität dieses Körpers zu gewinnen. Er zeigte wieder dieselben Eigenschaften, wie die früher beschriebenen. Er war unlöslich in Wasser, Alkalien, Säuren, Alkohol, Äther, Chloroform, Ligroin, Benzol, Petroläther. Es gelang nicht, aus ihm irgend welche charakterisierte chemische Verbindung zu gewinnen.

Dagegen zeigte die braune Flüssigkeit, die sich in dem Kathodenraum gebildet hatte, interessante Eigenschaften. Es glang, aus der filtrierten, klaren Flüssigkeit durch Zusatz von Kalilauge einen gelbbraunen krümeligen Körper zu fällen. Dieser Körper, der abgesaugt, mit kaltem Wasser ausgewaschen und getrocknet wurde, erwies sich als unlöslich in kaltem und heifsem Wasser, in Alkohol, Äther, Benzol, Chloroform, Pyridin. Löslich war er in der heifsen Lösung von Pyridinsulfat, ebenso in heifser konzentrierter Säure, besonders Schwefelsäure. Infolge dieser Eigenschaft wurde der Körper als Base oder Gemisch mehrerer Basen angesprochen. Ein Teil der Base wurde versuchsweise in das leicht lösliche schwefelsaure Salz verwandelt, mit Kalilauge die Base ausgefällt, und versucht, dieselbe, oder wenigstens ihren Hauptbestandteil mit Wasserdämpfen überzutreiben. Dies gelang aber nicht. Aus dem Filtrat der Base gelang es noch durch festes Kali, etwas abzuscheiden, doch war die Menge der zuerst erhaltenen gegenüber sehr unbedeutend. Der Rest des Filtrates wurde destillirt. Im Destillat fand sich Pyridin, mit minimalen Mengen Piperidin vermischt.

Versuch VII. Es wurden wieder Bleielektroden verwandt. Abweichend von den vorigen Versuchen wurde so geschaltet, dafs die aufserhalb der Thonzelle befindliche Bleiplatte von ca. $125 \mathrm{qcm}$ wirksamer Oberfläche Kathode war. Die Stromdichte $\mathrm{ND}_{100}$ wurde ungefähr $=8 \mathrm{Amp}$. genommen. Zur Vermeidung zu starker Erwärmung wurde mit kaltem Wasser gekühlt. Beobachtet wurde die Stromstärke $J$, die Badspannung $E$, die Temperatur $t$. 


\begin{tabular}{c|c|c|l}
\hline \hline Zeit & $J$ & $E$ & $t$ \\
\hline \hline $4^{12}$ & 10.3 & 7.1 & $42^{0}$ \\
$4^{18}$ & 10.3 & 6.75 & $49^{0}$ \\
$4^{25}$ & 10.3 & 6.5 & $52^{0}$ \\
$4^{30}$ & 10.6 & 6.5 & $54^{0}$ \\
$4^{40}$ & 10.6 & 6.75 & $57^{0}$ \\
$4^{50}$ & 10.0 & 8.5 & $59^{0}$ \\
5 & 9.3 & 13.25 & $62.5^{0}$ \\
$5^{10}$ & 9.0 & 13.5 & $63^{0}$
\end{tabular}

Als Resultat ergab sich wieder der braune Körper. Aus der abfiltrierten Kathodenflüssigkeit wurde wiederum die Base, hier als etwas hellerer flockiger Niederschlag abgeschieden. Die Reaktionen, welche sie nach dem Trocknen gab, waren die gleichen. Beide Körper wurden im Exsiccator über Schwefelsäure aufbewahrt: hierbei wurde ihre Färbung dunkler, ihr Volum kleiner, dafür aber ihre Konsistenz fester. Sie verbreiteten einen merkwürdigen Geruch, der vielleicht dem des Coniins etwas ähnlich war.

Versuch VIII. Die Bedingungen wurden wie bei den vorigen Versuchen gewählt. Gekühlt wurde mit Eis. Der Versuch begann um $11^{35}$ und wurde über fast drei Stunden ausgedehnt. Die dabei angestellten Beobachtungen waren die folgenden:

\begin{tabular}{c|l|r|l}
\hline \hline Zeit & $J$ & $E$ & $t$ \\
\hline \hline & & & \\
$11^{38}$ & 9.7 & 6.6 & $15^{0}$ \\
$11^{46}$ & 9.1 & 6.0 & $28^{0}$ \\
$11^{54}$ & 9.7 & 6.1 & $34^{0}$ \\
$12^{6}$ & 9.7 & 6.1 & $39^{0}$ \\
$12^{18}$ & 9.0 & 6.1 & $41.5^{0}$ \\
$12^{23}$ & 9.0 & 6.2 & $40^{0}$ \\
$12^{38}$ & 9.6 & 6.7 & $42^{0}$ \\
$12^{53}$ & 9.65 & 7.1 & $42.5^{0}$ \\
$1^{2}$ & 9.6 & 8.7 & $44^{0}$ \\
$1^{16}$ & 9.5 & 10.8 & $49.5^{0}$ \\
$1^{35}$ & 9.2 & 13.5 & $56.5^{0}$ \\
$1^{42}$ & 9.2 & 12.8 & $58^{0}$ \\
$1^{49}$ & 8.8 & 17.1 & $64^{0}$ \\
$2^{2}$ & 8.6 & 17.5 & $68^{\circ}$ \\
$2^{7}$ & 7.4 & 19.0 & $69^{\circ}$ \\
$2^{13}$ & 7.0 & 21.2 & $67^{0}$ \\
$2^{25}$ wurde ausgeschaltet. &
\end{tabular}


Die Resultate waren die gleichen, wie bei den vorhergehenden Versuchen. Auch hier wurde aus der Kathodenflüssigkeit die Base ausgefällt. Von der Ansicht ausgehend, dals der gebildete gelbbraune Körper aufser der Base noch Verunreinigungen enthalte, wurde versucht, denselben zu reinigen. Es wurde zu diesem Zweck ein lösliches Salz, z. B. das Sulfat oder Acetat dargestellt. Durch Alkali fiel der Körper wieder im gleichen Habitus aus. Das Auswaschen des Körpers gestaltete sich sehr schwierig: bei Anwendung einer Witr'schen Saugplatte trat bald Verstopfung der Poren des Papiers ein; ebenso gelang die Filtration durch Glaswolle sehr schlecht. Es wurde daher zur Entfernung des gebildeten Kalisalzes häufig mit heilsem Wasser ausgekocht und dieses abdekantiert.

Eine Reinigung der Base gelang auch nicht, wenn die Lösung des Salzes mit Tierkohle gekocht wurde; es wurde dadurch nicht der geringste Fortschritt erzielt. Ebenso wenig gelang es, durch andere Salze oder Trennungsmethoden den erhaltenen Körper zu reinigen oder zu zerlegen.

Versuch IX wurde unter den gleichen Bedingungen wie der vorhergehende ausgeführt; das Resultat war absolut analog. Die ausgefällte Base wurde in ihr lösliches salzsaures Salz übergeführt, und nun versucht, zu ihrer Charakterisierung Doppelsalze herzustellen. Durch Platinchlorid, Pikrinsäure, Quecksilberchlorid, gelbes Blutlaugensalz wurden in der Lösung ganz leicht starke Niederschläge, charakteristisch gefärbt, erzielt. Die Verbindungen, die in Lösung waren, zeigten beim Trocknen sehr starke Neigung zur Verharzung. Es dürften Verbindungen von sehr hoch zusammengesetzten Polymeren des Pyridins oder eines anderen Körpers der Reihe sein. Als letztes Mittel wurde der Weg gewählt, die Base selbst zu analysieren. Sie wurde im Achatmörser leicht zu einem braunen, etwas glänzenden Pulver verrieben, und eine Verbrennung sowie eine Stickstoffbestimmung ausgeführt. Die erhaltenen Resultate führten zu der Zusammensetzung $\mathrm{C}_{5} \mathrm{H}_{7} \mathrm{NO}$.

\begin{tabular}{lrc} 
& Gefunden: & Berechnet: \\
C & $61.70 \%$ & $61.86 \%$ \\
H & $\mathbf{7 . 5 1} \%$ & $7.22 \%$ \\
N & $13.93 \%$ & $14.43 \%$ \\
O (Diff.) $16.86 \%$ & $16.49 \%$ \\
\hline \multicolumn{2}{c}{$100.00 \%$} & $100.00 \%$
\end{tabular}

Da der analysierte Körper zweifellos Verunreinigungen enthielt, kann die berechnete Formel nicht als sicher hingestellt werden. 
Versuch X. Die Anordnung war die gleiche, wie bei den früheren Versuchen, die Kathode umgab rings die Thonzelle, in der sich die Anode befand und hatte eine der letzteren zugekehrte Oberfläche von $350 \mathrm{qcm}$. Die Konzentrationsbedingungen waren die von Ahrens angegebenen. Der Versuch wurde während 8 Tagen ununterbrochen im Gange erhalten. Den Strom lieferten vier hintereinandergeschaltete Akkumulatoren. Die Stromstärke betrug im Anfang 5 Amp., sank aber am Ende bis auf 1.8 Amp. $\mathrm{ND}_{100}$ war also im Mittel $=1 \mathrm{Amp}$. Die Spannung fiel von 4.9 auf 3.3 Volt. Die Gasentwickelung, welche gleich anfangs auftrat, blieb während der Dauer des Versuches mälsig stark; die Temperatur hielt sich ziemlich konstant.

Bei diesem Versuche bildete sich der braune Körper an der Kathode nicht, ebenso wenig konnte aus der Flüssigkeit durch Kalilauge eine Base ausgefüllt werden. Zur Untersuchung auf Piperidin wurde ein Teil der Kathodenflüssigkeit mit Benzoylchlorid versetzt. Es schieden sich Krystalle einer Benzoylverbindung aus. Diese wurden in $\ddot{t}$ ther aufgenommen und die Lösung im Scheidetrichter zu wiederholten Malen mit verdünnter Säure, Kalilauge und Wasser gewaschen. Aus der ätherischen Lösung schieden sich beim Verdunsten Krystalle aus, welche die Bildung einer sekundären Base des Piperidins oder eines seiner Derivate anzeigten. Doch war ihre Menge im Vergleich zum angewandten Flüssigkeitsquantum so gering, dafs nur ein kleiner Teil des Pyridins durch die Elektrolyse reduziert worden war.

Im allgemeinen entsprechen also meine Resultate durchaus nicht den Angaben von AmRens. Piperidin erhielt ich bei meinen Versuchen gar nicht oder nur in geringer Menge, während AHRens $95 \%$ der Theorie erhielt. Der braune Körper, der sich bei den Versuchen von AHrENs nur zweimal bildete und den dieser vergeblich wiederherzustellen suchte, zeigte sich bei mir nach jeder Elektrolyse (mit Ausnahme der letzten, wo mit sehr geringer Stromdichte gearbeitet wurde); es scheint jedoch nicht möglich zu sein, aus ihm ein falsbares Produkt zu isolieren. Aufserdem gelang es mir, aus der Kathodenflüssigkeit eine Base auszufällen, die AHRENs gar nicht erwähnt. Ferner stimmen meine Versuche in mehreren unwesentlicheren Angaben, wie Temperatur, Gasentwickelung, mit den Arraess'schen nicht überein. Auch indem ich mich von den Bedingungen, die nach den Angaben von Amrens leicht zu Fehlern Veranlassung geben können, möglichst entfernte, konnte ich seine 
Resultate nicht erreichen. Es scheint also, dals bei den Aurens'schen Versuchen gewisse nicht näher untersuchte Umstände obwalteten, von deren Vorhandensein wohl AHRENs selbst kaum Kenntniss hatte, da er in seinen Arbeiten nichts darüber angiebt. Versuch XI. Da es möglich ist, mit Hilfe des elektrischen Stromes Salpetersäure bei Anwesenheit von Metall zu Ammoniak zu reduzieren (es beruht darauf eine quantitativ analytische Methode zur Bestimmung der Salpetersäure ${ }^{1}$ ) machte ich mit Pyridin einen ähnlichen Versuch, in der Hoffnung, Piperidin zu erhalten. Zur Elektrolyse wurde das Doppelsalz des Pyridinnitrates mit Kupfernitrat benutzt. Als Zersetzungsgefäfs diente eine CruAssen'sche Platinschale, die spiralförmige Anode befand sich zur Sicherheit in einem Thoncylinder, der in der Mitte der Schale stand. $\mathrm{ND}_{\mathbf{1 0 0}}$ war $=0.5$ Amp., die Dauer der Elektrolyse 14 Stunden. Das Kupfer schied sich schlecht und schwammig ab. Die Temperatur stieg auf 55 $5^{\circ}$, die Gasentwickelung war mälsig. Das Resultat war ein negatives; es konnte in der Zersetzungsflüssigkeit kein Reduktionsprodukt des Pyridins nachgewiesen werden.

Vorstehende Arbeit wurde im S.-S. 1896 und im W.-S. 1896/97 im elektrochemischen Laboratorium der $\mathrm{Kgl}$. Techn. Hochschule Berlin-Charlottenburg auf Anregung des Herrn Prof. Dr. G. v. KnorRe ausgeführt.

Es ist mir eine angenehme Pflicht, meinem hochverehrten Lehrer für das Interesse, welches er mir bei Anfertigung dieser Arbeit entgegengebracht hat, an dieser Stelle meinen herzlichsten Dank auszusprechen.

1 Vortmann, Ber. deutsch. chem. Ges. 23, 2798.

Bei der Redaktion eingegangen am 29. März 1897. 